Pacific Journal of Mathematics

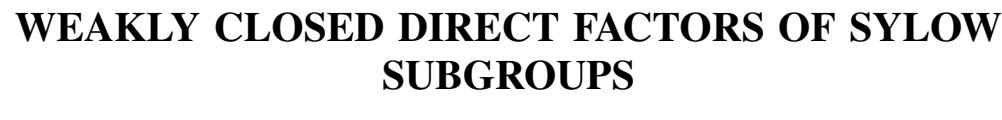




\section{WEAKLY CLOSED DIRECT FACTORS OF SYLOW SUBGROUPS}

\section{G. Glauberman and J. G. Thompson}

In many finite classical linear groups and permutation groups, certain Sylow subgroups have weakly closed direct factors. In this paper we establish a sufficient condition for this to occur in arbitrary finite groups.

The purpose of this paper is to prove the following result:

THeorem A. Let $p$ be an odd prime, and let $P$ be a Sylow $p$ subgroup of a finite group $G$. Suppose $Q$ and $R$ are subgroups of $G$ such that $P=Q \times R$. Assume that no indecomposable factor of $R$ is isomorphic to a subgroup of $Q$. Then $P$ contains a weakly closed direct factor that is isomorphic to $R$.

Our notation is taken from [3]. In addition, for every finite $p$ group $P$, we let

$$
d(P)=\max .\{|A| \mid A \text { is an Abelian subgroup of } P\}
$$

and

$$
J(P)=\langle A| A \text { an Abelian subgroup of } P \text { and }|A|=d(P)\rangle .
$$

The following lemma is a special case of a result of Wielandt (Satz 6 of [9]).

Lemma 1. Let $A$ and $B$ be subgroups of a finite group $G$ such that $G=A B$. Suppose $p$ is a prime, $A_{p}$ is a normal $p$-subgroup of $A$, and $B_{p}$ is a normal $p$-subgroup of $B$. Then $\left\langle A_{p}, B_{p}\right\rangle$ is a p-group.

Proof. By Sylow's Theorem, $\left\langle\left(A_{p}\right)^{g}, B_{p}\right\rangle$ is a $p$-group for some $g \in G$. Take $a \in A$ and $b \in B$ such that $a b=g$. Then $\left(A_{p}\right)^{g}=\left(\left(A_{p}\right)^{a}\right)^{b}=$ $\left(A_{p}\right)^{b}$. Also, $\left(B_{p}\right)^{b^{-1}}=B_{p}$. Thus

$$
\left\langle A_{p}, B_{p}\right\rangle=\left\langle\left(A_{p}\right)^{a},\left(B_{p}\right)^{b^{-1}}\right\rangle=\left\langle\left(A_{p}\right)^{q}, B_{p}\right\rangle^{b^{-1}},
$$

which is a $p$-group.

An automorphism $\alpha$ of a group $G$ is said to be central if $g^{\alpha} g^{-1} \in Z(G)$ for all $g \in G$. We say that an element (or a subgroup) of Aut $G$ fixes a subgroup $H$ of $G$ if it (or its elements) map $H$ onto $H$.

THEOREM 1. Let $\pi$ be a set of primes and $G$ be a finite $\pi$-group. 
Suppose $G=H \times K$ and no indecomposable factor of $H$ is isomorphic to an indecomposable factor of $K$. Let $A=$ Aut $G$ and let $C$ be the group of central automorphisms of $G$. Then $G$ has the following properties:

(a) If $H^{*} \cong H, K^{*} \cong K$, and $G=H^{*} \times K^{*}$, then $G=H^{*} \times K=$ $H \times K^{*}$.

(b) The groups $H \times Z(K), Z(H) \times K, H^{\prime}$, and $K^{\prime}$ are characteristic subgroups of $G$.

(c) There exists a normal, nilpotent $\pi$-subgroup $D$ of $A$ that is contained in $C$ and permutes transitively the pairs $\left(H^{*}, K^{*}\right)$ such that

$$
H^{*} \cong H, K^{*} \cong K \text {, and } G=H^{*} \times K^{*} \text {. }
$$

(d) If $B$ is a $\pi^{\prime}$-subgroup of $A$ then there exists a pair $\left(H^{*}, K^{*}\right)$ such that

$$
H^{*} \cong H, K^{*} \cong K, G=H^{*} \times K^{*},
$$

and $B$ fixes $H^{*}$ and $K^{*}$. Moreover, if $B$ fixes $H$, we may take $H^{*}=H$.

Proof. (a) Represent $H$ and $K$ as products of indecomposable factors, say, $H=H_{1} \times \cdots \times H_{r}$ and $K=K_{1} \times \cdots \times K_{s}$. Then $G=$ $H \times K=H_{1} \times \cdots \times H_{r} \times K_{1} \times \cdots \times K_{s}$. Since $H^{*} \cong H$ and $K^{*} \cong K$, we have a similar representation

$$
G=H^{*} \times K^{*}=H_{1}^{*} \times \cdots \times H_{r}^{*} \times K_{1}^{*} \times \cdots \times K_{s}^{*} .
$$

Obviously, there exists a one-to-one correspondence $\phi$ between the factors $F$ of the first representation and those of the second representation. By the Krull-Schmidt Theorem [7, p. 81], $\phi$ may be chosen to have the properties that $\phi(F) \cong F$ for each $F$ and

$$
G=\phi\left(H_{1}\right) \times \cdots \times \phi\left(H_{r}\right) \times K_{1} \times \cdots \times K_{s} .
$$

Clearly, for every $H_{i}, \phi\left(H_{i}\right)$ is some $H_{j}^{*}$. Hence $G=H^{*} \times K$. By symmetry, $G=H \times K^{*}$.

(b) Let $\alpha \in A$. Then $G=H^{a} \times K^{\alpha}$. By (a), $G=H^{\alpha} \times K$. Thus

$$
(\boldsymbol{C}(K))^{\alpha}=(H \times \boldsymbol{Z}(K))^{\alpha} \subseteq H^{\alpha} \boldsymbol{Z}(G) \subseteq \boldsymbol{C}(K) .
$$

Hence $H \times Z(K)$ is a characteristic subgroup of $G$. Since $H^{\prime}=$ $(H \times Z(K))^{\prime}, H^{\prime}$ is also a characteristic subgroup of $G$. By symmetry, $Z(H) \times K$ and $K^{\prime}$ are characteristic in $G$.

(c) For each $\alpha \in C$, define $\alpha-1$ by $g^{\alpha-1}=g^{-1} g^{\alpha}$ for all $g \in G$. Since $\alpha \in C, \alpha-1$ is an endomorphism of $G$ and $G^{\alpha-1} \subseteq Z(G)$. Thus $g^{\alpha-1}=g^{\alpha} g^{-1}$ for all $g \in G$. 
Let $D_{H}$ be the group of all $\alpha \in C$ for which $g^{\alpha}=g$ for all $g \in H$ and $g^{\alpha-1} \in \boldsymbol{Z}(H)$ for all $g \in G$. Then

$$
H^{\alpha-1}=1 \text { and } G^{\alpha-1} \subseteq Z(H), \text { for } \alpha \in D_{H} .
$$

Define $D_{K}$ similarly.

Suppose $\alpha \in D_{H}$. Let $\eta=\alpha-1$. Take $g \in G$, and let $h=g^{\eta}$. By (1), it is clear by induction that

$$
g^{\alpha^{i}}=g h^{i} \quad \text { for } \quad i=1,2,3, \cdots .
$$

Thus

(2) the order of $\alpha$, the exponent of $G^{\alpha-1}$, and the exponent of $G / \operatorname{Ker}(\alpha-1)$ are equal.

We also observe from (1) that if $\alpha, \beta \in D_{H}$, then $\alpha \beta=\beta \alpha$. Thus (3) $D_{H}$ is an Abelian $\pi$-group.

Suppose $\alpha \in D_{H}, \beta \in D_{K}$, and $\alpha$ and $\beta$ have relatively prime orders. Let $g \in G$, and let $h=g^{\alpha-1}$ and $k=g^{\beta-1}$. Then $h \in Z(H)$ and $k \in Z(K)$. By (2), the order of $h$ divides the order of $\alpha$. Since an analogue of (2) also holds for elements of $D_{K}, h \in \operatorname{Ker}(\beta-1)$. Similarly, $k \in \operatorname{Ker}(\alpha-1)$. Hence

$$
g^{\alpha \beta}=\left(g^{\alpha}\right)^{\beta}=(g h)^{\beta}=g^{\beta} h^{\beta}=g^{\beta} h=g k h=g h k
$$

and

$$
g^{\beta \alpha}=\left(g^{\beta}\right)^{n}=(g k)^{\alpha}=g^{\alpha} k^{\alpha}=g^{\alpha} k=g h k=g^{\alpha \beta} .
$$

Thus $\alpha \beta=\beta \alpha$. In particular, if $p$ and $q$ are distinct primes, (4) the Sylow $p$-subgroup of $D_{H}$ centralizes the Sylow $q$-subgroup of $D_{K}$.

$$
\text { Suppose } \begin{gathered}
H^{*} \cong H, K^{*} \cong K, \text { and } G=H^{*} \times K^{*} . \text { By (a), } \\
G=H \times K=H \times K^{*}=H^{*} \times K .
\end{gathered}
$$

Define a mapping $\eta: G \rightarrow G$ as follows: For each $k \in K$, take $h^{\prime} \in H$ and $k^{*} \in K^{*}$ such that $k=h^{\prime} k^{*}$. Let $k^{\eta}=h^{\prime}$. For $h \in H$ and $k \in K$, let

$$
(h k)^{\eta}=k^{\eta} \text {. }
$$

Then $\eta$ is an endomorphism of $G$. Since $K$ and $K^{*}$ centralize $H$, $G^{\eta}=K^{\natural} \leqq Z(H) \subseteq Z(G)$. Hence the mapping $\alpha: G \rightarrow G$ given by $g^{\alpha}=$ $\left(g^{\gamma}\right)^{-1} g$ is an endomorphism of $G$. Since $H^{\alpha}=H$ and $K^{\alpha}=K^{*}, \alpha$ is an automorphism of $G$. Clearly, $\alpha \in D_{H}$. Thus $D_{H}$ permutes transitively all the direct factors of $G$ that are isomorphic to $K$. Similarly $D_{K}$ permutes transitively all the direct factors of $G$ that are isomorphic to $H$.

Let $A_{H}$ be the set of all $\alpha \in A$ such that $H^{\alpha}=H$. Define $A_{K}$ similarly. Then 


$$
D_{H} \triangleleft A_{H} \text { and } D_{K} \triangleleft A_{K} \text {. }
$$

Let $\alpha \in A$. Then $H^{\alpha} \cong H, K^{\alpha} \cong K$, and $G=H^{\alpha} \times K^{\alpha}$. Hence there exists $\beta \in D_{H}$ such that $K^{\beta}=K^{\alpha}$. Therefore $K^{\alpha^{\beta}-1}=K$, and $\alpha \beta^{-1} \in A_{K}$. Thus $\alpha \in A_{K} A_{H}$. So

$$
A=A_{K} A_{H}=A_{H} A_{K} \text {. }
$$

Let $I=A_{H} \cap A_{K}$, and take $\alpha \in A_{H}$. As in the previous paragraph, there exists $\beta \in D_{H}$ such that $K^{\alpha}=K^{\beta}$. Thus $\alpha \beta^{-1} \in A_{H} \cap A_{K}=I$. So $A_{H}=I D_{H}=D_{H} I$. Similarly, $A_{K}=I D_{K}=D_{K} I$.

Let $p$ be a prime. By (5), $O_{p}\left(D_{H}\right)$ is a normal subgroup of $A_{H}$ and $\boldsymbol{O}_{p}\left(D_{K}\right)$ is a normal subgroup of $A_{K}$. Let $D_{p}=\left\langle\boldsymbol{O}_{p}\left(D_{H}\right), \boldsymbol{O}_{p}\left(D_{K}\right)\right\rangle$. By (5), (6), and Lemma $1, D_{p}$ is a $p$-group. By (3) and (4), every $p^{\prime}$-element in $D_{H}$ or $D_{K}$ centralizes $D_{p}$. Since $D_{p}$ normalizes itself, $D_{H}$ and $D_{K}$ normalize $D_{p}$. Since $I$ normalizes $D_{H}$ and $D_{K}, I$ normalizes $D_{p}$. Hence

$$
N\left(D_{p}\right) \supseteqq\left\langle D_{H}, D_{K}, I\right\rangle=\left\langle D_{H} I, D_{K} I\right\rangle=A_{H} A_{K}=A .
$$

Let $D$ be the subgroup of $C$ generated by the groups $D_{p}$ for all primes $p$. Then $D_{H} \subseteq D$ and $D_{K} \subseteq D$, by (3). Suppose $H^{*} \cong H$, $K^{*} \cong K$, and $G=H^{*} \times K^{*}$. Then there exists $\alpha \in D_{K}$ and $\beta \in D_{H}$ such that $H^{* \alpha}=H$ and $\left(\left(K^{*}\right)^{\alpha}\right)^{\beta}=K$. Now $\alpha \beta \in D, H^{* \alpha \beta}=H$, and $K^{* \alpha \beta}=K$. This completes the proof of (c).

(d) Retain the notation of (c). Then $I=A_{H} \cap A_{K}$ and $A=I D$. Since $D \leqq B D \cong A=I D, B D=(B D \cap I) D$. Note that $D$ is nilpotent and $|B|$ and $|D|$ are relatively prime. By Schur's Theorem $[10, \mathrm{p}$. 162], $B D \cap I$ splits over $D \cap I$. Let $B^{*}$ be a complement of $D \cap I$ in $B D \cap I$. Thus $B^{*}$ is a complement of $D$ in $B D$. By the SchurZassenhaus Theorem [10, p. 162], $B^{*}$ is conjugate to $B$ in $B D$. Take $\alpha \in B D$ such that $B=\alpha^{-1} B^{*} \alpha$. Since $B^{*} \subseteq A_{H} \cap A_{K}, B$ fixes $H^{\alpha}$ and $K^{\alpha}$.

If $B$ fixes $H$, then $B \subseteq A_{H}=I D_{H}$. An argument similar to the previous one shows that $\alpha B \alpha^{-1} \subseteq I$ for some $\alpha \in B D_{H}$. Then $B$ fixes $H^{\alpha}$ and $K^{\alpha}$, and $H^{\alpha}=H$. This completes the proof of Theorem 1.

Lemma 2. Let $p$ be a prime and $P$ be a p-subgroup of a finite group $G$. Suppose $H$ is a $p^{\prime}$-subgroup of $G$ that normalizes $P$. Then:

(a) $P=[P, H] C_{P}(H)$;

(b) $[[P, H], H]=[P, H]$; and

(c) if $P$ is Abelian, then $P=[P, H] \times C_{P}(H)$.

Proof. This result is well known. Parts (a) and (b) appear as Corollary 3 of Theorem 1 of [4]. Part (c) follows directly from part (a) and from the lemma on page 172 of [10]. 
Lemma 3. Let $p$ be a prime and $P$ be a p-subgroup of a finite group $G$. Suppose $H$ is a $p^{\prime}$-subgroup that normalizes $P$. Assume that

(a) $P$ is Abelian and $H$ centralizes $\Omega_{1}(P)$ or that

(b) $P$ has no Abelian direct factors and $H$ centralizes $P / Z(P)$. Then $H$ centralizes $P$.

Proof. (a) By Lemma 2, $P=[P, H] \times C_{P}(H)$. Hence $\Omega_{1}([P, H])=1$. Therefore, $[P, H]=1$, i.e., $H$ centralizes $P$.

(b) Let $Q=[P, H]$. Then $Q \subseteq Z(P)$, so $Q$ is Abelian. By Lemma 2, $P=Q C_{P}(H), Q=[Q, H]$, and $Q \cap C_{P}(H)=[Q, H] \cap C_{Q}(H)=1$. Since $Q \subseteq Z(P), C_{P}(H) \triangleleft P$. Hence $P=Q \times C_{P}(H)$. By $(\mathrm{b}), Q=1$.

Lemma 4. Let $P$ and $Q$ be normal Abelian p-subgroups of $a$ finite group $G$. Suppose that $Q \subseteq P$ and that some Sylow p-subgroup of $G$ normalizes some complement of $Q$ in $P$. Then $G$ normalizes some complement $R$ of $Q$ in $P$.

Proof. By constructing a semi-direct product if necessary, we may assume that $G$ is a splitting extension of $P$ by a group $E$ that is isomorphic to $G / C(P)$. Let $S$ be a Sylow $p$-subgroup of $E$. Then $S$ normalizes some complement $R^{*}$ of $O$ in $P$. Now, $S P$ is a Sylow $p$-subgroup of $G$ and $S R^{*}$ is a complement of $Q$ in $S P$. Thus $S P$ splits over $Q$. By a theorem of Gaschütz [6, p. 246], $G$ splits over $Q$. Let $C$ be a complement of $Q$ in $G$, and let $R=C \cap P$.

The following result is a special case of a theorem of Wielandt (Satz 12, page 193, of [8]).

Lemma 5. Suppose $p$ is a prime and $P$ is a Sylow p-subgroup of a finite group $G$. Let $n=|N(P) / P|$. Let $V$ be the transfer of $G$ into $P / P^{\prime}$.

(a) If $a \in P \cap Z(N(P))$ and $a^{p}=1$, then $V(a)=a^{n} P^{\prime}$.

Furthermore, suppose $P^{\prime} \subseteq Q \subseteq P$ and suppose $W$ is the transfer of $G$ into $P / Q$. Then:

(b) If $A \subseteq P \cap Z(N(P))$ and $A \cap Q=1$, then $A \cap G^{\prime}=A \cap \operatorname{Ker} W=1$.

(c) If $Q \triangleleft N(P)$, then $\Omega_{1}(Q \cap Z(P)) \subseteq \operatorname{Ker} W$.

Proof. (a) Let $r=|G: P|$, and let $P x_{i}, i=1,2, \cdots, r$, be the distinct cosets of $P$ in $G$. We may assume that

$$
\begin{gathered}
x_{1}, \cdots, x_{n} \in N(P) ; P x_{i} a=P x_{i}(1 \leqq i \leqq s) \\
P x_{i} a \neq P x_{i}(s+1 \leqq i \leqq r)
\end{gathered}
$$


where $s \geqq n$. Since $a^{p}=1$, Lemma 14.4.1, page 206, of [6] yields

$$
V(a)=P^{\prime} \prod_{i \leqq i \leqq s} x_{i} a x_{i}^{-1}
$$

Since $a \in Z(N(P))$,

$$
V(a)=P^{\prime} a^{n} \prod_{n<i \leqq s} x_{i} a x_{i}^{-1}
$$

Suppose $x \in P$ and $n<i \leqq s$. Then $\left(P x_{i}\right) x=P x_{j}$ for some $j$. Since

$$
P x_{j} a=P x_{i} x a=P x_{i} a x=P x_{i} x=P x_{j}
$$

and since $x_{i} \notin N(P), n<j \leqq s$. Thus $P$ permutes the cosets $P x_{i}$, $n<i \leqq s$, by right multiplication. We may assume that $P x_{n+1}, \cdots, P x_{t}$ are representatives of the distinct orbits of $P$. For $i=n+1, \cdots, t$, let $P_{i}$ be the subgroup of $P$ fixing $P x_{i}$, and let $y_{i 1}, \cdots, y_{i_{i}}$ be representatives of the distinct left cosets of $P_{i}$ in $P$. Then the orbit of $P x_{i}$ is $P x_{i} y_{i j}, 1 \leqq j \leqq m_{i}$.

Suppose $n+1 \leqq i \leqq t$. Since $x_{i} \notin N(P), P x_{i} P \neq P x_{i} \quad$ Thus $P_{i} \subset P$ and

$$
m_{i} \equiv\left|P: P_{i}\right| \equiv 0 \text {, modulo } p \text {. }
$$

We may assume that, for $k=n+1, \cdots, s$, every $x_{k}$ has the form $x_{i} y_{i j}$ for some (unique) $i$ and $j$. By (7) and (8),

$$
\begin{aligned}
V(a) & =P^{\prime} a^{n} \prod_{n<i \leqq t} \prod_{1 \leqq j \leqq m_{i}} x_{i} y_{i j} a y_{i j}^{-1} x_{i}^{-1} \\
& =P^{\prime} a^{n} \prod_{n<i \leqq t}\left(x_{i} a x_{i}^{-1}\right)^{m_{i}}=P^{\prime} a^{n},
\end{aligned}
$$

as desired.

(b) Suppose $a \in A$ and $a^{p}=1$. Now, $W$ is simply the composition of $V$ with the natural mapping of $P / P^{\prime}$ into $P / Q$. Hence $W(a)=$ $a^{n} Q$, by (a). Since $p$ does not divide $n$ and since $a \notin Q, W(a) \neq Q$. Thus $A \cap \operatorname{Ker} W$ has no elements of order $p$, so $A \cap \operatorname{Ker} W=1$. Since $G^{\prime} \subseteq \operatorname{Ker} W, A \cap G^{\prime}=1$.

(c) Let $B=\Omega_{1}(Q \cap Z(P))$ and $N=N(P)$. Since $N / C_{N}(B)$ is a $p^{\prime}$ group,

$$
B=[B, N] \times C_{B}(N),
$$

by Lemma 2. Obviously, $[B, N] \leqq G^{\prime} \cong \operatorname{Ker} W$. Let $a \in C_{B}(N)$. From (a),

$$
W(a)=\left(a^{n} P^{\prime}\right) Q=a^{n} Q=Q,
$$

so $a \in \operatorname{Ker} W$. Thus $B \subseteq \operatorname{Ker} W$. This completes the proof of Lemma 5 . 
We now require the following proposition, which is the main result of [5]:

Theorem 2. Let $p$ be an odd prime, and let $P$ be a Sylow $p$ subgroup of a finite group $G$. Suppose $x \in P \cap Z(N(J(P)))$. Then $g^{-1} x g=x$ whenever $g \in G$ and $g^{-1} x g \in P$.

THEOREM 3. Let $p$ be a prime, and let $P$ be a Sylow p-subgroup of a finite group $G$. Suppose $Q$ and $R$ are normal subgroups of $N(P)$ and $P=Q \times R$. Assume that $R \leqq O_{p}(G)$ and that no indecomposable direct factor of $R$ is isomorphic to a subgroup of $Q$. Then $R^{\prime}$ is a normal subgroup of $G$, and there exists a normal subgroup $R^{*}$ of $G$ such that $P=Q \times R^{*}$. Moreover, if $p$ is odd and $R / R^{\prime}$ is a normal subgroup of $N_{G / R^{\prime}}\left(J\left(P / R^{\prime}\right)\right)$, we may take $R^{*}=R$.

Proof. Let $Q_{1}=\boldsymbol{O}_{p}(G) \cap Q$. Since $R \cong \boldsymbol{O}_{p}(G) \subseteq P=R \times Q, \boldsymbol{O}_{p}(G)=$ $R \times Q_{1}$. Now, no indecomposable factor of $R$ is isomorphic to an indecomposable factor of $Q_{1}$. By Theorem $1, R Z\left(Q_{1}\right)$ and $R^{\prime}$ are characteristic subgroups of $O_{p}(G)$ and are therefore normal subgroups of $G$.

Let $T=R Z\left(Q_{1}\right)=Z\left(Q_{1}\right) \times R$. Represent $R$ as a direct product of an Abelian subgroup $R_{a}$ and a subgroup $R_{b}$ having no Abelian direct factors. By Theorem 1 , we may assume that $R_{a}$ and $R_{b}$ are normalized by a complement of $P$ in $N(P)$ and are therefore normal in $N(P)$. If $R_{a} \neq 1$, let $p^{e}$ be the minimum of the exponents of the indecomposable factors of $R_{a}$. If $R_{a}=1$, let $p^{e}=p|T|$. Then let

$$
T_{0}=\left\langle x^{p^{e-1}} \mid x \in T\right\rangle \text {. }
$$

Now $T_{0} \triangleleft G$ and

$$
\Omega_{1}\left(R_{a}\right) \subseteq T_{0} \subseteq R
$$

Since $Q$ centralizes $R, Q$ centralizes $T_{0}$ and $T / Z(T)$. Let

$$
C=\boldsymbol{C}_{G}(T / \boldsymbol{Z}(T)) \cap \boldsymbol{C}_{G}\left(T_{0}\right) \text { and } H=C T .
$$

Then $C$ and $H$ are normal in $G$ and $P=Q R \subseteq C T=H$.

Let $K$ be a complement of $P$ in $N_{H}(P)$. Since $H / C \cong T /(C \cap T)$, $K \subseteq C$. Thus $[T, K] \subseteq Z(T)$ and $K$ centralizes $T_{0}$. Therefore $\left[R_{b}, K\right] \subseteq Z\left(R_{b}\right)$ and, by $(9), K$ centralizes $\Omega_{1}\left(R_{a}\right)$. By Lemma $3, K$ centralizes $R_{a}$ and $R_{b}$. So $K$ centralizes $R$.

Let $\bar{H}=H / R^{\prime}, \bar{R}=R / R^{\prime}, \bar{K}=K R / \bar{R}^{\prime}$, and so forth. Then $\bar{R} \leqq Z(\bar{P})$ and $N_{\bar{H}}(\bar{P})=\bar{P} \bar{K}$, so

$N_{\bar{H}}(\bar{P})$ centralizes $\bar{R}$. 
Let $W$ be the transfer of $\bar{H}$ into $\bar{P} / \bar{Q}$. By Lemma $5(\mathrm{~b})$,

$$
\bar{R} \cap \bar{H}^{\prime} \subseteq \bar{R} \cap \operatorname{Ker} W=1 \text {. }
$$

By the Frattini argument,

$$
G=H N(P) \text {. }
$$

Suppose $p$ is odd and $\bar{R} \triangleleft N_{\bar{G}}(J(\bar{P}))$. Then by (11)

$$
\left[\bar{R}, N_{\bar{H}}(J(\bar{P}))\right] \subseteq \bar{R} \cap \bar{H}^{\prime}=1 .
$$

Thus by Theorem 2 no element of $\bar{R}$ is conjugate to any other element of $\bar{P}$. Since $\bar{R} \subseteq O_{p}(\bar{G}) \subseteq \bar{P}$, we must have $\bar{R} \subseteq Z(\bar{H})$. Therefore, $R \triangleleft H$. By (12) $R$ is normal in $G$, as claimed.

Let us return to the general case. Now, $\bar{P}=\bar{Q} \times \bar{R}$. By (11), $\bar{R} \cap \operatorname{Ker} W=1$. Since

$$
\mid \text { Image }(W)|\leqq| \bar{P} / \bar{Q}|=| \bar{R} \mid,
$$

$\bar{R}$ is a complement to $\operatorname{Ker} W$ in $\bar{H}$. Hence $\bar{R}$ is a complement to $\bar{T} \cap \operatorname{Ker} W$ in $\bar{T}$. Since $W$ depends only on $\bar{H}$ and $\bar{Q}$ and since $N(P)$ normalizes $H$ and $Q, N(P)$ normalizes $\operatorname{Ker} W$. By (12), $\bar{G}$ normalizes Ker $W$. Hence $\bar{T} \cap$ Ker $W \triangleleft \bar{G}$. Now $\bar{T}^{\prime}=\bar{R}^{\prime}=1$ and $\bar{P}$ normalizes $\bar{R}$. By Lemma 4 , there exists a complement $\bar{R}^{*}$ of $\bar{T} \cap \operatorname{Ker} W$ in $\bar{T}$ such that $\bar{R}^{*} \triangleleft \bar{G}$. Let $R^{*}$ be the subgroup of $T$ that contains $R^{\prime}$ and satisfies $R^{*} / R^{\prime}=\bar{R}^{*}$.

By Lemma $5, \Omega_{1}(\boldsymbol{Z}(\bar{Q})) \leqq \operatorname{Ker} W$. Since $\Omega_{1}(\boldsymbol{Z}(Q)) R^{\prime} / R^{\prime} \subseteq \Omega_{1}(\boldsymbol{Z}(\bar{Q}))$, (11) yields

$$
\Omega_{1}(Z(Q)) \cap R^{*} \sqsubseteq \Omega_{1}(Z(Q)) \cap R^{\prime} \subseteq Q \cap R=1 .
$$

Hence $Q \cap R^{*}$ is normal in $Q$ but intersects $Z(Q)$ in 1 , so $Q \cap R^{*}=1$. Consequently, $\left|Q R^{*}\right|=|Q|\left|R^{*}\right|=|Q||R|=|P|$. Since $Q, R^{*} \triangleleft P$, $P=Q \times R^{*}$. This completes the proof of Theorem 3 .

We now require the following concepts and results of Alperin and Gorenstein ( $\S 2$ of [2] and $\S 5$ of [1]):

Definition. Let $G$ be a finite group and $p$ be a prime. Let $\mathscr{H}$ be the set of all nonidentity $p$-subgroups of $G$. A conjugacy functor $W$ on $\mathscr{H}$ is a mapping from $\mathscr{H}$ into $\mathscr{H}$ that satisfies the following two conditions for each $H$ in $\mathscr{H}$ :

(a) $\boldsymbol{W}(H) \subseteq H$

(b) $\boldsymbol{W}\left(H^{x}\right)=W(H)^{x}$ for all $x \in G$.

Theorem 4. Let $p$ be a prime and $P$ be a nonidentity Sylow p-subgroup of a finite group $G$. Let $W$ be a conjugacy functor on the set of nonidentity p-subgroups of $G$. Then there exists a class 
of nonidentity subgroups of $P$, called well-placed subgroups, having the following properties:

(1) If $H$ is a well-placed subgroup then $N(H) \cap P$ is a Sylow p-subgroup of $\boldsymbol{N}(H)$, and $\boldsymbol{W}(\boldsymbol{N}(H) \cap P)$ is a well-placed subgroup.

(2) Suppose $R \subseteq P, g \in G$, and $R^{g} \subseteq P$. Then there exists a sequence of well-placed subgroups $H_{1}, \cdots, H_{n}$ and elements $x_{1}, \cdots, x_{n}$ of $G$ such that

(a) $g=x_{1} \cdots x_{n}$,

(b) $x_{i} \in N\left(H_{i}\right), 1 \leqq i \leqq n$, and

(c) $R \cong H_{1}$ and $R^{x_{1} \cdots x_{i}} \subseteq H_{i+1}, 1 \leqq i \leqq n-1$.

Theorem 4 easily yields the following result:

Corollary. Let $p$ be a prime and $P$ be a Sylow p-subgroup of a finite group $G$. Suppose $Q \subseteq P$ and $Q$ is not weakly closed in $P$ with respect to $G$. Then there exists $H \subseteq P$ and $g \in N(H)$ such that $H$ is well-placed, $Q \subseteq H$, and $Q^{g} \neq Q$.

Theorem 5. Let $p$ be a prime, and let $P$ be a Sylow p-subgroup of a finite group $G$. Suppose $P=Q \times R$ and no indecomposable direct factor of $R$ is isomorphic to a subgroup of $Q$. Let $J$ be the subgroup of $P$ that contains $R^{\prime}$ and satisfies $J / R^{\prime}=J\left(P / R^{\prime}\right)$. Then

(a) There exists $R^{*} \triangleleft N(J)$ such that $P=Q \times R^{*}$.

(b) If $p$ is odd and $R^{*}$ satisfies (a), $R^{*}$ is weakly closed in $P$ with respect to $G$.

Proof. (a) Let $K$ be a complement of $P$ in $N(P)$. By Theorem 1 , we may assume that $K$ normalizes $Q$ and $R$. Hence $Q, R \triangleleft N(P)$. Since $R / R^{\prime} \subseteq Z\left(P / R^{\prime}\right)$,

$$
R \subseteq J \subseteq \boldsymbol{O}_{p}(\boldsymbol{N}(J))
$$

Thus, (a) follows from Theorem 3.

(b) Assume $p$ is odd and $R^{*}$ satisfies (a) but is not weakly closed in $P$. We may assume that $R=R^{*}$. By a theorem of Burnside [6, p. 46], there exists a subgroup $P_{0}$ of $P$ such that $P_{0} \supseteq R$ and $R \pitchfork N\left(P_{0}\right)$. Since

$$
R \sqsubseteq P_{0} \subseteq P=R \times Q, \quad P_{0}=R \times\left(P_{0} \cap Q\right) .
$$

By Theorem 1 and our hypothesis on $Q$ and on $R, R^{\prime} \triangleleft N\left(P_{0}\right)$. Therefore, $R$ is not weakly closed in $P$ with respect to $N\left(R^{\prime}\right)$. Since $P \subseteq N(J) \subseteq N\left(R^{\prime}\right)$, we may assume that $R^{\prime} \triangleleft G$.

We define a conjugacy functor $W$ on the set of nonidentity subgroups $H$ of $G$ as follows: 


$$
W(H)=H, \quad \text { if } \quad R^{\prime} \nsubseteq H \text {; }
$$

and

$$
R^{\prime} \leqq W(H) \quad \text { and } \quad \boldsymbol{W}(H) / R^{\prime}=\boldsymbol{J}\left(H / R^{\prime}\right), \quad \text { if } \quad R^{\prime} \cong H .
$$

By the Corollary of Theorem 4, there exists a well-placed subgroup $H$ of $G$ having the properties that $H \supseteqq R$ and $R \rtimes N(H)$. Choose $H$ such that $P \cap N(H)$ has maximal order subject to these conditions. Let $P_{1}=P \cap N(H)$. Since $H$ is well-placed, $P_{1}$ is a Sylow $p$-subgroup of $N(H)$. By Theorem 3, $R / R^{\prime} \pitchfork N_{G / R^{\prime}}\left(J\left(P_{1} / R^{\prime}\right)\right)$. Hence $P_{1} \subset P$ by (a). But $J\left(P_{1} / R^{\prime}\right)=W\left(P_{1}\right) / R^{\prime}$. Thus $R \subseteq P_{1}$ and $R \unlhd \boldsymbol{N}\left(\boldsymbol{W}\left(P_{1}\right)\right)$. Since $H$ is well placed and $P_{1} \subset P, W\left(P_{1}\right)$ is well placed and

$$
P_{1} \subset P \cap N\left(P_{1}\right) \subseteq P \cap N\left(W\left(P_{1}\right)\right) .
$$

But this contradicts the choice of $H$. Thus we have proved Theorem 5. Theorem A obviously follows from Theorem 5 .

REMARK. Let $A^{n}$ and $S^{n}$ be the alternating and symmetric groups of degree $n$, for $n=4,6$. Since Theorem 2 holds for $p=2$ when $S^{4}$ is not involved in $G$ [5], Theorem A holds for $p=2$ when $S^{4}$ is not involved in $N\left(R^{\prime}\right) / R^{\prime}$.

Let $H=A^{6}$, and let $R$ be an indecomposable 2-group of order greater than eight. Take a transposition $\tau$ in $S^{6}$ and a subgroup $R_{0}$ of index two in $R$. Consider $R$ as an operator group on $H$ by defining $h^{r}=h$ when $r \in R_{0}$ and $h^{r}=\tau^{-1} h \tau$ when $r \in R$ and $r \notin R_{0}$. Let $G$ be the semi-direct product of $H$ by $R$, and embed $H$ and $R$ in $G$ in the natural manner. Then $C_{I I}(R)$ contains a Sylow 2-subgroup $Q$ of $H$. Let $P=Q \times R$. Then $P$ is a Sylow 2-subgroup of $G$ and $R$ is not isomorphic to any subgroup of $Q$, but $P$ has no weakly closed direct factor isomorphic to $R$.

\section{REFERENCES}

1. J. Alperin, Sylow intersections and fusion, J. Algebra 6 (1967), 222-241.

2. J. Alperin and D. Gorenstein, Transfer and fusion in finite groups, J. Algebra 6 (1967), 242-255.

3. W. Feit and J. G. Thompson, Solvability of groups of odd order, Pacific J. Math. 13 (1963), 775-1029.

4. G. Glauberman, Fixed points in groups with operator groups, Math. Z. 84 (1964), $120-125$.

5. - Weakly closed elements of Sylow subgroups, Math. Z. (to appear).

6. M. Hall, The Theory of Groups, Macmillan, New York, 1959.

7. J. Rotman, The Theory of Groups: An Introduction, Allyn and Bacon, Boston, 1965.

8. H. Wielandt, $p$-Sylowgruppen and p-Faktorgruppen, J. reine angew. Math. 182 (1940), 180-193. 
9. Uber das Product paarweise vertauschbarer nilpotenter Gruppen, Math. Z. 55 (1951), 1-7.

10. H. Zassenhaus, The Theory of Groups, Second English Edition, Chelsea, New York, 1958.

Received May 12, 1967.

UNIVERSity OF ChicAgo 



\section{PACIFIC JOURNAL OF MATHEMATICS}

\section{EDITORS}

\author{
H. ROYDEN \\ Stanford University \\ Stanford, California \\ R. R. Phelps \\ University of Washington \\ Seattle, Washington 98105
}

J. DUGUNDJI

Department of Mathematics

University of Southern California

Los Angeles, California 90007

RICHARD ARENS

University of California

Los Angeles, California 90024

\section{ASSOCIATE EDITORS}
E. F. BECKENBACH
B. H. NeumanN
F. WOLF
K. YosidA

\section{SUPPORTING INSTITUTIONS}

UNIVERSITY OF BRITISH COLUMBIA

CALIFORNIA INSTITUTE OF TECHNOLOGY

UNIVERSITY OF CALIFORNIA

MONTANA STATE UNIVERSITY

UNIVERSITY OF NEVADA

NEW MEXICO STATE UNIVERSITY

OREGON STATE UNIVERSITY

UNIVERSITY OF OREGON

OSAKA UNIVERSITY

UNIVERSITY OF SOUTHERN CALIFORNIA
STANFORD UNIVERSITY

UNIVERSITY OF TOKYO

UNIVERSITY OF UTAH

WASHINGTON STATE UNIVERSITY

UNIVERSITY OF WASHINGTON

AMERICAN MATHEMATICAL SOCIETY CHEVRON RESEARCH CORPORATION TRW SYSTEMS

NAVAL WEAPONS CENTER

Printed in Japan by International Academic Printing Co., Ltd., Tokyo, Japan 


\section{Pacific Journal of Mathematics}

\section{Vol. 26, No. $1 \quad$ November, 1968}

Efraim Pacillas Armendariz, Closure properties in radical theory......... 1

Friedrich-Wilhelm Bauer, Postnikov-decompositions of functors .......... 9

Thomas $\mathrm{Ru}-$ Wen Chow, The equivalence of group invariant positive definite

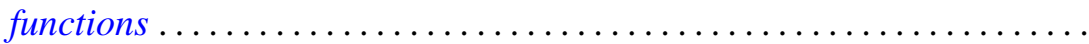

Thomas Allan Cootz, A maximum principle and geometric properties of

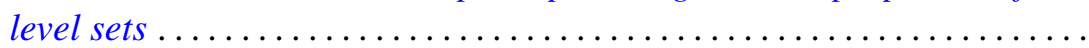

Rodolfo DeSapio, Almost diffeomorphisms of manifolds ............ 47

R. L. Duncan, Some continuity properties of the Schnirelmann density......

Ralph Jasper Faudree, Jr., Automorphism groups of finite subgroups of

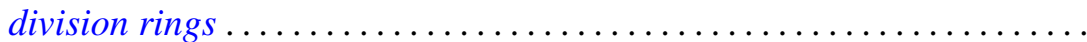

Thomas Alastair Gillespie, An invariant subspace theorem of $J$.

Feldman.........................................

George Isaac Glauberman and John Griggs Thompson, Weakly closed direct factors of Sylow subgroups .............................

Hiroshi Haruki, On inequalities generalizing a Pythagorean functional equation and Jensen's functional equation .....................

David Wilson Henderson, D-dimension. I. A new transfinite dimension.....

David Wilson Henderson, D-dimension. II. Separable spaces and

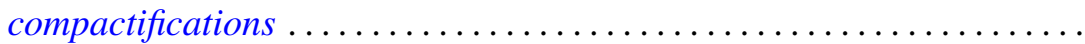

Julien O. Hennefeld, A note on the Arens products ............... 115

Richard Vincent Kadison, Strong continuity of operator functions ...

J. G. Kalbfleisch and Ralph Gordon Stanton, Maximal and minimal coverings of $(k-1)$-tuples by $k$-tuples.

Franklin Lowenthal, On generating subgroups of the Moebius group by pairs of infinitesimal transformations...

Michael Barry Marcus, Gaussian processes with stationary increments possessing discontinuous sample paths . .

Zalman Rubinstein, On a problem of Ilyeff ...

Bernard Russo, Unimodular contractions in Hilbert space. ...

David Lee Skoug, Generalized Ilstow and Feynman integrals...

William Charles Waterhouse, Dual groups of vector spaces . 